\title{
Phononic soft mode behavior and a strong electronic background across the structural phase transition in the excitonic insulator $\mathrm{Ta}_{2} \mathrm{NiSe}_{5}$
}

\author{
Min-Jae Kim $\odot,{ }^{1,2, *}$ Armin Schulz $\odot,{ }^{1}$ Tomohiro Takayama, ${ }^{1,3}$ Masahiko Isobe, ${ }^{1}$ Hidenori Takagi, ${ }^{1,3,4}$ and Stefan Kaiser $\odot^{1,2, \dagger}$ \\ ${ }^{1}$ Max Planck Institute for Solid State Research, 70569 Stuttgart, Germany \\ ${ }^{2} 4$ th Physics Institute, University of Stuttgart, 70569 Stuttgart, Germany \\ ${ }^{3}$ Institute for Functional Matter and Quantum Technologies, University of Stuttgart, 70569 Stuttgart, Germany \\ ${ }^{4}$ Department of Physics, University of Tokyo, Tokyo 113-0033, Japan
}

(Received 10 July 2020; revised 30 October 2020; accepted 11 November 2020; published 10 December 2020)

\begin{abstract}
$\mathrm{Ta}_{2} \mathrm{NiSe}_{5}$ became one of the most investigated candidate materials for hosting an excitonic insulator ground state. Many studies describe the corresponding phase transition as a condensation of excitons breaking a continuous symmetry. This view got challenged recently pointing out the importance of the loss of two mirror symmetries at a structural phase transition that occurs together with the semiconductor-excitonic insulator transition. For such a scenario an unstable optical zone-center phonon at low energy is proposed to drive the transition. Here we report on the experimental observation of such a soft mode behavior using Raman spectroscopy. In addition we find a novel spectral feature, likely of electronic or joint electronic and phononic origin, that is clearly distinct from the lattice dynamics and that becomes dominant at $T_{c}$. This suggests a picture of joint structural and electronic orders driving the phase transition.
\end{abstract}

DOI: 10.1103/PhysRevResearch.2.042039

Introduction. Recently the possibility of realizing the elusive state of an excitonic insulator (EI) in the zero-gap semiconductor $\mathrm{Ta}_{2} \mathrm{NiSe}_{5}$ has stimulated a tremendous body of experimental and theoretical work. An EI consists of condensed excitons forming a new phase of matter whose proposed macroscopic quantum states may resemble many properties of superconductors and superfluids [1-8]. Besides this interesting test bed for many-body physics, the ultrafast response in $\mathrm{Ta}_{2} \mathrm{NiSe}_{5}$ under laser excitation is a potential platform for novel optoelectronic applications $[9,10]$. An EI transition by spontaneous condensation of excitions is expected in narrow bandwidth semimetals or small band-gap semiconductors if the exciton binding energy exceeds the bandwidth or the band gap of the system. EIs have been realized in specifically designed electron-hole bilayer systems [11-15] or by creating high exciton densities under strong photoexcitation [16-18]. However, identifying fingerprints of EI phases in bulk materials remains an experimental challenge. Prominent candidates, such as $1 T-\mathrm{TiSe}_{2}$ show an indirect band gap, and, therefore, the potential EI state appears together with a charge density wave (CDW) that seems to drive the dynamics $[19,20]$. In contrast, $\mathrm{Ta}_{2} \mathrm{NiSe}_{5}$ is a direct band-gap semiconductor that has been proposed to show

\footnotetext{
*mj.kim@fkf.mpg.de

†s.kaiser@fkf.mpg.de
}

Published by the American Physical Society under the terms of the Creative Commons Attribution 4.0 International license. Further distribution of this work must maintain attribution to the author(s) and the published article's title, journal citation, and DOI. Open access publication funded by the Max Planck Society. an EI transition without charge or other finite momentum order [21,22]. A semiconductor-insulator transition appears at $T_{c}=328 \mathrm{~K}$ that can be tuned via chemical or physical pressure [23,24]. Evidence for excitonic condensation comes from a characteristic band flattening seen in angle-resolved photoemission [25-27] and the opening of a gap in electronic $[9,28]$ and optical [29-31] spectra. Further fingerprints stem from nonequilibrium responses of the ground state that are compatible with the melting and relaxation dynamics of a condensate [32-36] and the excitation of possible collective modes of a coherent ground state [37-41]. Nevertheless, the origin of the gap is still under debate due to a structural phase transition from a high-temperature orthorhombic to a lowtemperature monoclinic phase that happens simultaneously at $T_{c}$ despite the absence of a CDW $[22,42] . \mathrm{Ta}_{2} \mathrm{NiSe}_{5}$ crystallizes as a quasi-one-dimensional structure of Ta-Ni-Ta chains. In the low-temperature phase two mirror symmetries of the high-temperature phase are broken by a shear motion of the $\mathrm{Ta}$ against the Ni chains [Fig. 1, inset]. Using inelastic $\mathrm{x}$-ray measurements Nakano et al. [42] show that this displacement can be decomposed into $B_{2 g}$ phonons of the high-temperature phase. Although they find a significant frequency jump of the transverse acoustic mode describing the Se atom motion when approaching the phase transition from the high-temperature side, they cannot evidence a softening in the optical phonon mode in the temperature range they accessed. The latter mode describes the Ta atom shear motion that happens at the phase transition. But a strong linewidth broadening of this mode indicates strong electron phonon coupling. A strong lattice interaction is also evidenced by strong Fano resonances [30] and polaronic bands [31] in optics or the lattice coupled dynamics of the nonequilibrium responses [32,33,36-41]. The proposed scenario for the $\mathrm{EI}$ in $\mathrm{Ta}_{2} \mathrm{NiSe}_{5}$ is that the excitonic 


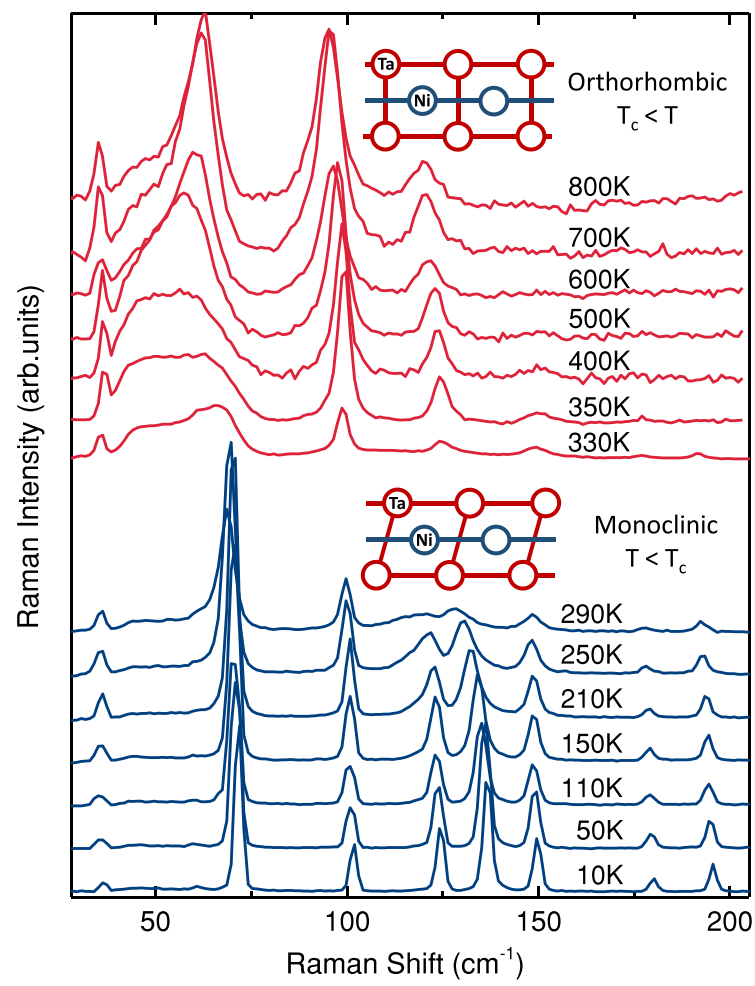

FIG. 1. Raman spectrum of $\mathrm{Ta}_{2} \mathrm{NiSe}_{5}$ in the orthorombic phase above (red) and in the monoclinic phase below (blue) the structural phase transition at $T_{c}=328 \mathrm{~K}$. At the phase transition the Ta chains perform a shear displacement with respect to the Ni chain. The corresponding crystal structures are shown as insets. Spectra are taken in $(X-)$ geometry revealing the $A_{g}$ channel below and $A_{g}+B_{2 g}$ channel above $T_{c}$.

condensate couples to the lattice phonons and as such drives a joint excitonic and structural instability $[24,29,43]$. However, this picture of exciton condensation driven by pure electronic Coulomb interactions of the electron hole pairs got recently challenged. Exciton condensation would require breaking a continuous symmetry and a complex order parameter to describe the low-temperature phase of $\mathrm{Ta}_{2} \mathrm{NiSe}_{5}$. In particular Mazza et al. [44] and Watson et al. [45] point out the loss of two mirror symmetries in the low-temperature phase are linked to a discrete symmetry break. They propose possible excitonic (linearly coupled to lattice modes) and structural instabilities that are in agreement with the experimental observations of Nakano et al. [42]. Both instabilities lead to a hybridization of the $\mathrm{Ta}$ and $\mathrm{Ni}$ bands in the center of the Brillouin zone in contrast to the pure bands in a condensation scenario. A theoretical density functional theory study by Subedi [46] has recently explored the corresponding zone center optical phonon branches relevant for this case and suggests that the optical $B_{2 g}$ modes could drive the phase transition. According to the calculations, if the $B_{2 g}$ phonon mode softens as the $T_{c}$ is approached from above; the dynamical instability is due to an unstable optical phonon mode. No softening should be observed if the instability is electronic or due to an unstable elastic mode corresponding to a uniform shear distortion of the lattice. However, clear experimental fingerprints for the dynamics of such instabilities are missing.
This motivates our Raman studies on $\mathrm{Ta}_{2} \mathrm{NiSe}_{5}$ presented here to explore the crucial low-frequency behavior. We clearly identify a $B_{2 g}$ soft mode above $T_{c}$ and characterize a broad electronic or coupled electronic-phononic feature next to the soft mode that dominates across the phase transition.

Experiment. Single crystals of $\mathrm{Ta}_{2} \mathrm{NiSe}_{5}$ were grown by chemical vapor transport reaction as described in Ref. [23]. Raman measurements are performed within specific excitation geometries and parameters as detailed in the Supplemental Material [47].

Results. Figure 1 shows the Raman spectra of $\mathrm{Ta}_{2} \mathrm{NiSe}_{5}$ in $Y(X-) \bar{Y}$ geometry in the temperature range from 10 to $800 \mathrm{~K}$. That goes clearly beyond the $400 \mathrm{~K}$ covered in the inelastic X-ray measurements [42]. The blue spectra are taken in the monoclinic phase below $T_{c}$ and belong in the given experimental geometry to the $A_{g}$ channel. Above $T_{c}$ spectra are shown in red and are taken in the orthorhombic phase so that in this geometry the experiment probes the $A_{g}$ and $B_{2 g}$ channels. Spectra below $T_{c}$ show clear peaks at 101, 124, 136, 149, 180, and $195 \mathrm{~cm}^{-1}$ identified as $A_{g}$ symmetries already in earlier studies $[9,48]$. On increasing temperature the high-frequency modes do not show strong changes up to $T_{c}$ except the modes at 124 and $136 \mathrm{~cm}^{-1}$ shifting to lower energy and broadening. At the same time they decrease in amplitude. Above $T_{c}$ the $124-\mathrm{cm}^{-1}$ mode disappears whereas the $136-\mathrm{cm}^{-1}$ mode shows up to the highest temperature. Increasing the temperature further shows a slight redshift and broadening of the mode. The $101-\mathrm{cm}^{-1}$ mode above $T_{c}$ shows a similar trend. This behavior is known from the previous studies [48]. Here we will focus, in particular, on the so far unexplored low-frequency response with prominent peaks at 36 and $71 \mathrm{~cm}^{-1}$ below $T_{c}$. The $36-\mathrm{cm}^{-1} A_{g}$ mode is known as a peculiar mode that under strong impulsive excitation shows a coupling to the excitonic system and resembles amplitude mode behavior [37]. In agreement with reports in the nonequilibrium study, in the equilibrium measurements this mode shows basically no change in width or shift in frequency as function of temperature. In amplitude the equilibrium mode increases as the function of increasing temperature in stark contrast to the nonlinear excitation.

$A_{g}$ and $B_{2 g}$ low-frequency spectra. However the $71-\mathrm{cm}^{-1}$ mode and the spectral range right below will become the dominant key features of the transition. Below $T_{c}$, in the monoclinic phase, shown as the blue spectra in Fig. 1, the mode barely changes on increasing temperature except for a small redshift on approaching $T_{c}$. Hardly noticeable but already present below $T_{c}$ is a small broad background of spectral weight in the frequency range from 42 to $71 \mathrm{~cm}^{-1}$. Its weight increases towards $T_{c}$. Above $T_{c}$ in the orthorhombic phase, shown in the red spectra, very prominent changes happen. Across the phase transition the mode at $71 \mathrm{~cm}^{-1}$ is strongly suppressed, and the broad spectral feature becomes clearly visible next to this mode. On further temperature increase in the orthorhombic phase, the broad spectral feature is most prominent between 330 and $400 \mathrm{~K}$ before an additional peak appears on top of the broad background and becomes sharper and dominant on increasing temperature.

To understand and characterize this broad feature as well as the appearing modes we map each symmetry channel individually (Supplemental Material S2 [47]). Both key features, 


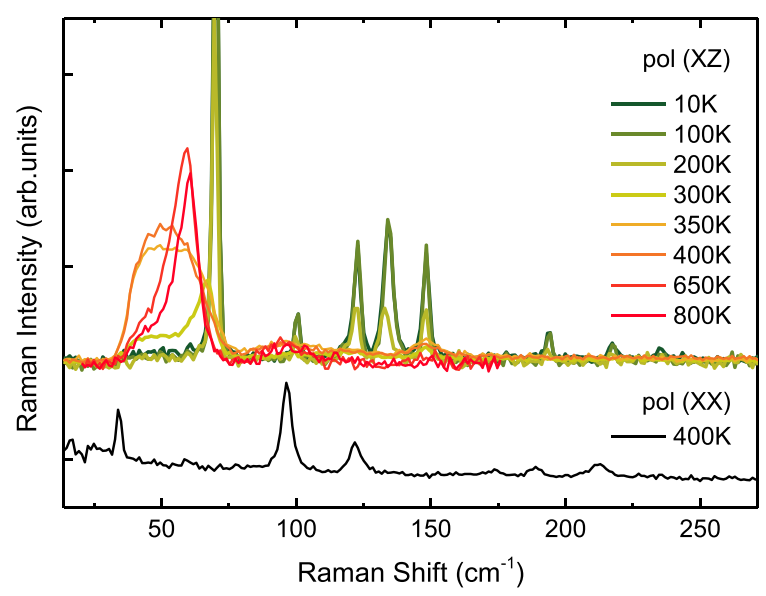

FIG. 2. Temperature-dependent Raman spectra in the (XZ) geometry probing the $A_{g}$ channel below $T_{c}$ and the $B_{2 g}$ channel above $T_{c}$. The $A_{g}$ channel above $T_{c}$ is shown for $400 \mathrm{~K}$ in (XX) geometry.

the $71-\mathrm{cm}^{-1} A_{g}$ mode and the broad new feature, appear only in the XZ channel. We plot the temperature-dependent spectra of this channel in Fig. 2. Below $T_{c}$ (green spectra), probing the $A_{g}$ channel we find the sharp phonon modes at $71 \mathrm{~cm}^{-1}$ and the high-frequency modes at 101, 124, 136, 149, 180, and $195 \mathrm{~cm}^{-1}$. On increasing temperature the $A_{g}$ modes in the XZ configuration are suppressed and disappear at $T_{c}$. This is in fully understood since XZ probes the $B_{2 g}$ channel above $T_{c}$. The $A_{g}$ modes remain in the XX configuration at $400 \mathrm{~K}$ that probes the $A_{g}$ channel above $T_{c}$ (black spectrum). In the $B_{2 g}$ channel of the XZ geometry above $T_{c}$ (orange/red spectra) new small modes appear at 96 and $150 \mathrm{~cm}^{-1}$. Most prominent is the appearance of the broad background feature at temperatures up to $400 \mathrm{~K}$ and the additional new peak around $60 \mathrm{~cm}^{-1}$ on further temperature increase.

Characterization of the modes. In the following we present the fit of the temperature dependence of the spectra. All phonon modes are fit using single Gaussian functions. However the anomalous broad spectral feature is difficult to capture. It can be fit using multiple Gaussians (where the number of fit parameters becomes large) or as we have performed here using a super Gaussian function. Figure 3 shows the significant low-frequency Raman spectra in the XZ geometry and the extracted fit parameters for three features: (1) As a key feature at highest temperatures we identify a strong sharp $B_{2 g}$ phonon mode at $60 \mathrm{~cm}^{-1}$ (blue). On decreasing temperature this mode clearly softens to $52 \mathrm{~cm}^{-1}$ and significantly broadens approaching $T_{c}$. Its intensity decreases and disappears at $T_{c}$. This soft mode we classify as the $B_{2 g}$ zone-center optical phonon mode predicted by Subedi [46]. The inelastic X-ray study [42] did not find this unstable $B_{2 g}$ mode in the range of 328-400 K since it already softened and broadened significantly. Also within our data we cannot resolve the mode below $400 \mathrm{~K}$ where the mode becomes buried under the strong response of a broad spectral feature that is discussed below. (2) However, at $400 \mathrm{~K}$ we see the onset of a new mode that evolves into the $71-\mathrm{cm}^{-1}(\sim 2-\mathrm{THz}) A_{g}$ mode in the monoclinic phase below $T_{c}$ (green) where the mode suddenly sharpens at the phase transition. In line with the interpretation of Subedi this new mode in the monoclinic phase corresponds to the amplitude modulation of the order parameter deriving from the unstable $B_{2 g}$ mode of the orthorhombic phase [46]. The most surprising feature in $\mathrm{Ta}_{2} \mathrm{NiSe}_{5}$ is (3) the anomalous broad spectral component that dominates the weight in the
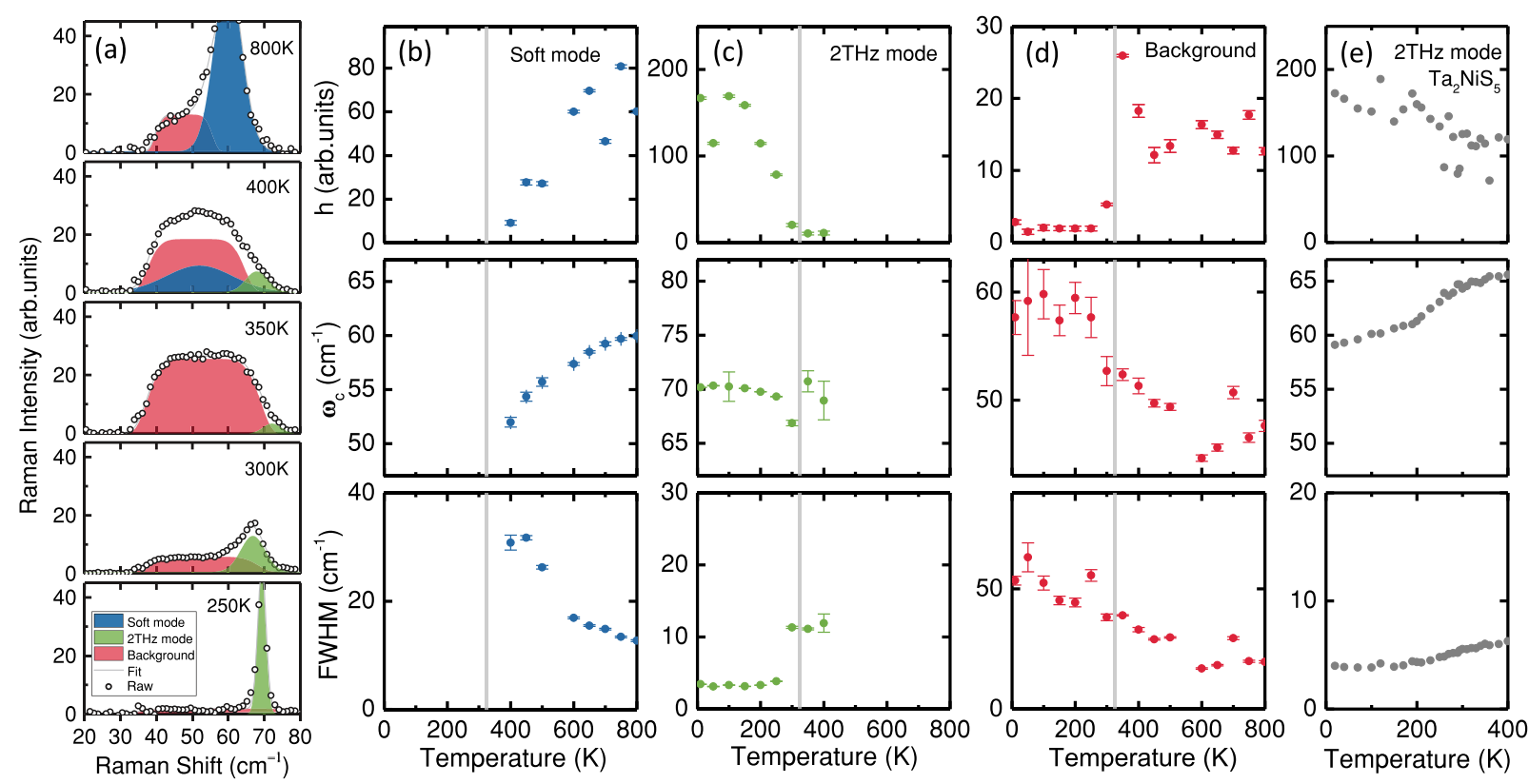

FIG. 3. (a) Fit to the low-frequency Raman dynamics of $\mathrm{Ta}_{2} \mathrm{NiSe}_{5}$ in the (XZ) channel. Exemplary spectra and extracted parameters of amplitude center frequency and width of the different modes (b)-(d). The key contributions are (b) zone-center $B_{2 g}$ soft phonon mode above $T_{c}$ (blue), and (c) a 2-THz $A_{g}$ mode (green) interpreted as amplitude modulation of the order parameter deriving from the unstable $B_{2 g}$ mode. In addition (d) a broad spectral feature (red) next to these modes appears across the phase transition with its intensity becoming dominant at $T_{c}$. For comparison the dynamics of the $2-\mathrm{THz}$ mode in $\mathrm{Ta}_{2} \mathrm{NiS}_{5}$ are given which does not undergo any phase transition (gray) in (e). 


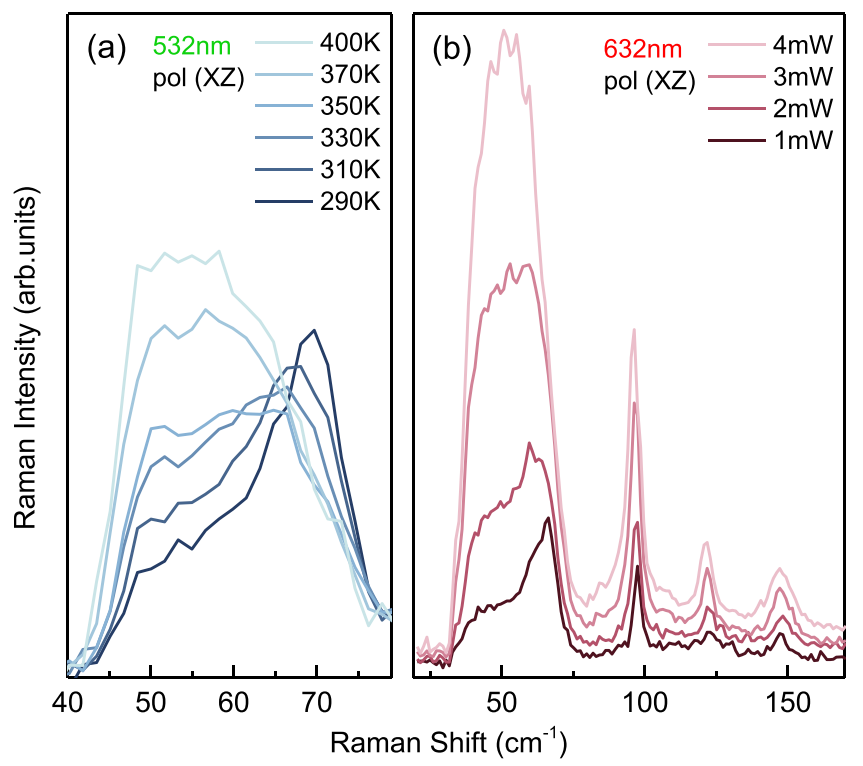

FIG. 4. Laser-dependent low-frequency Raman dynamics of $\mathrm{Ta}_{2} \mathrm{NiSe}_{5}$. (a) Temperature-dependent Raman spectra using 532-nm excitation wavelength. (b) Laser power-dependent Raman spectra at $300 \mathrm{~K}$ using 632-nm excitation. Spectra normalized to the increased fluence are shown in the Supplemental Material [47].

temperature range around the phase transition (red). At very high temperatures it clearly exists with a strong finite weight next to the soft mode. When cooling below $450 \mathrm{~K}$ its weight significantly increases, and it becomes the dominant feature approaching $T_{c}$. Then its intensity significantly drops at $T_{c}$ but remaining comparable to other modes even below $T_{c}$, as seen, e.g., at the 300-K data. Only when cooling further down the relative weight decreases further but remains finite down to lowest temperatures. As mentioned this feature is fit using a super-Gaussian contribution so that it is difficult to assign. Possibilities are fluctuations that are small in the low-temperature ordered phase that significantly increase and peak at $T_{c}$ and remain very prominent even for temperatures far above $T_{c}$. As the feature also could be fit with multiple peaks it also could represent other yet unidentified phonon modes that in the high-temperature phase are suppressed from the strong soft mode behavior. To gain additional information on the background feature we have performed the Raman measurement with different excitation conditions. Figure 4(a) shows that changing the excitation wavelength to $532 \mathrm{~nm}$ reproduces the results of Fig. 2 at $632 \mathrm{~nm}$. However, significant changes appear on heating the system with high laser power in the Raman experiment rather than using a thermal heater. The laser heating response in Fig. 4(b) reveals that the broad spectral feature is highly susceptible to the heating via laser fluence whereas the phononic system is not. This observation indicates an electronic or joint electronic-phononic origin of the feature that links to the proposed excitonic fluctuations. Important to note is that the soft mode behavior of the $B_{2 g}$ soft mode does not appear on laser heating. It is either fully buried in the electronic background or is suppressed as discussed in the Supplemental Material (S3) [47]. This observation points towards the important role of the electronic contribu- tion across the phase transition. However, its detailed role and coupling to the soft mode behavior need to be investigated in future studies.

To contrast the soft mode instability and the strong electronic background feature likely jointly driving the phase transition and the appearance of a new mode in the monoclinic low-temperature phase of $\mathrm{Ta}_{2} \mathrm{NiSe}_{5}$ we show the phonon mode behavior in the sister compound $\mathrm{Ta}_{2} \mathrm{NiS}_{5}$; a semiconductor that does not show an excitonic nor a structural phase transition [23]. Its equivalent 2-THz mode (Fig. 3, gray) shows a roughly monotonic increase in intensity and only a slight redshift on cooling across the measured temperature range. Also the mode stays always very sharp in the whole temperature range.

Conclusion. We have performed a detailed study of the low-frequency lattice dynamics across the orthorhombic to monoclinic structural phase transition in $\mathrm{Ta}_{2} \mathrm{NiSe}_{5}$. We can clearly identify a $B_{2 g}$ optical zone-center phonon soft mode in the orthorhombic phase at temperatures above $T_{c}$. This observation underlines a proposed mechanism by Subedi [46] that this instability could act as a prime cause for the structural phase transition; leading to the appearance of a new $A_{g}$ mode in the monoclinic phase below $T_{c}$, which we also clearly observe. However, this change between the different modes in their specific symmetry channels seems not to happen abruptly at $T_{c}$ but smeared out around the structural phase transition. Interestingly, right in that regime a broad new feature in the low-frequency regime dominates that traces fluctuating electronic or fluctuating joint electronic and structural order that drive the transition. Recently two similar Raman studies appeared as preprints $[49,50]$ that do not report any soft mode behavior but claim a pure excitonic driven phase transition based on a low-frequency electronic background. Our Rapid Communication does not show such a significant electronic background except the broad electronic feature discussed in Fig. 3. Our electronic feature also remains distinct if the data is Bose-factor corrected: As discussed in the Supplemental Material (S4) [47] reasons for the different response reported in Refs. [49,50] are possible polarization leaks from other crystallographic directions and most important the laser heating of the electronic system. As we have shown high laser power induces a dominant electronic heating and, therefore, stronger electronic background whereas the $B_{2 g}$ soft mode behavior is not observed. This heating of the electronic system also explains that high power pump probe experiments do not trigger the structural phase transition [32]. Here it explains why the two other Raman studies do not find the phononic soft mode as we report here and only see a dominant electronic response (see also Supplemental Material discussions S3 and S4 [47]). However, the studies [49,50] identify excitonic fluctuations as the nature of their electronic background. That substantiates our identification of the broad background feature being of dominant electronic origin. Excitonic fluctuations that we can link to the feature are strongest at $T_{c}$ as in Ref. [50]. However in our Rapid Communication we observe that they not solely drive an excitonic phase transition but smear out a joint structural and electronic transition. Their dominant contribution and potential to suppress the soft mode behavior points to their important role in driving the phase transition. 
From this we conclude that in $\mathrm{Ta}_{2} \mathrm{NiSe}_{5}$ the phase transition is neither purely phononic [45,51] nor purely or dominant electronic $[49,50,52]$ as from contradicting conclusions in various studies. The present Rapid Communication rather points to an interplay of both electronic and structural orders. The details of this interplay and the important question which contribution is the dominant driver cannot be solved simply by the static Raman study presented here alone. Future time-resolved studies may shed light on this problem by disentangling the two contributions. A possible scenario could be the self-localized exciton in polaron complexes as suggested in Refs. [30,31]. These may act as natural cavities for the excitonic system. This results in the observed high suscepti- bility of the electronic system to laser fluence by breaking the excitons and the only weakly affected lattice dynamics of the remaining polaronic distortion. This may also explain some of the contradicting conclusions drawn in the literature on the nature of the excitonic/structural phase transition in $\mathrm{Ta}_{2} \mathrm{NiSe}_{5}$ so far. As such the present Rapid Communication serves as a benchmark experimentally describing a jointly driven transition and as a trigger to further theoretical and experimental studies to characterize the electronic and phononic interplays in $\mathrm{Ta}_{2} \mathrm{NiSe}_{5}$.

Acknowledgments. We thank A. Subedi and A. V. Boris for insightful discussions.
[1] N. F. Mott, The transition to the metallic state, Philos. Mag. 6, 287 (1961).

[2] L. V. Keldysh and A. N. Kozlov, Collective properties of excitons in semiconductors, Sov. Phys. JETP 27, 521 (1968).

[3] D. Jerome, T. M. Rice, and W. Kohn, Excitonic insulator, Phys. Rev. 158, 462 (1967).

[4] B. I. Halperin and T. M. Rice, Possible anomalies at a semiconductor-semiconductor transition, Rev. Mod. Phys. 40, 755 (1968)

[5] F. X. Bronold and H. Fehske, Possibility of an excitonic insulator at the semiconductor-semiconductor transition, Phys. Rev. B 74, 165107 (2006).

[6] K. Seki, R. Eder, and Y. Ohta, BCS-BEC crossover in the extended Falicov-Kimball model: Variational cluster approach, Phys. Rev. B 84, 245106 (2011).

[7] B. Zenker, D. Ihle, F. X. Bronold, and H. Fehske, Electron-hole pair condensation at the semimetal-semiconductor transition: A BCS-BEC crossover scenario, Phys. Rev. B 85, 121102(R) (2012).

[8] Z. Sun and A. J. Millis, Bardasis-schrieffer polaritons in excitonic insulators, Phys. Rev. B 102, 041110(R) (2020).

[9] S. Y. Kim, Y. Kim, C.-J. Kang, E.-S. An, H. K. Kim, M. J. Eom, M. Lee, C. Park, T.-H. Kim, H. C. Choi, B. I. Min, and J. S. Kim, Layer-confined excitonic insulating phase in ultrathin $\mathrm{Ta}_{2} \mathrm{NiSe}_{5}$ Crystals, ACS Nano 10, 8888 (2016).

[10] L. Li, W. Wang, L. Gan, N. Zhou, X. Zhu, Q. Zhang, H. Li, M. Tian, and T. Zhai, Ternary $\mathrm{Ta}_{2} \mathrm{NiSe}_{5}$ flakes for a highperformance infrared photodetector, Adv. Funct. Mater 26, 8281 (2016).

[11] S. De Palo, F. Rapisarda, and G. Senatore, Excitonic Condensation in a Symmetric Electron-Hole Bilayer, Phys. Rev. Lett. 88, 206401 (2002).

[12] J. P. Eisenstein and A. H. MacDonald, Bose-Einstein condensation of excitons in bilayer electron systems, Nature (London) 432, 691 (2004).

[13] J. J. Su and A. H. MacDonald, How to make a bilayer exciton condensate flow, Nat. Phys. 4, 799 (2008).

[14] D. Nandi, A. D. K. Finck, J. P. Eisenstein, L. N. Pfeiffer, and K. W. West, Exciton condensation and perfect Coulomb drag, Nature (London) 488, 481 (2012).

[15] Perali, D. Neilson, and A. R. Hamilton, High-Temperature Superfluidity in Double-Bilayer Graphene, Phys. Rev. Lett. 110, 146803 (2013).
[16] D. Snoke, Spontaneous bose coherence of excitons and polaritons, Science 298, 1368 (2002).

[17] L. V. Butov, C. W. Lai, A. L. Ivanov, A. C. Gossard, and D. S. Chemla, Towards bose-einstein condensation of excitons in potential traps, Nature (London) 417, 47 (2002).

[18] L. V. Butov, A. C. Gossard, and D. S. Chemla, Macroscopically ordered state in an exciton system, Nature (London) 418, 751 (2002).

[19] J. A. Wilson, Concerning the semimetallic characters of $\mathrm{TiS}_{2}$ and $\mathrm{TiSe}_{2}$, Solid State Commun. 22, 551 (1977).

[20] H. Cercellier, C. Monney, F. Clerc, C. Battaglia, L. Despont, M. G. Garnier, H. Beck, P. Aebi, L. Patthey, H. Berger, and L. Forró, Evidence for an Excitonic Insulator Phase in $1 T-\mathrm{TiSe}_{2}$, Phys. Rev. Lett. 99, 146403 (2007).

[21] S. A. Sunshine and J. A. Ibers, Structure and physical properties of the new layered ternary chalcogenides tantalum nickel sulfide $\left(\mathrm{Ta}_{2} \mathrm{NiS}_{5}\right)$ and tantalum nickel selenide $\left(\mathrm{Ta}_{2} \mathrm{NiSe}_{5}\right)$, Inorg. Chem. 24, 3611 (1985).

[22] F. J. Di Salvo, C. H. Chen, R. M. Fleming, J. V. Waszczak, R. G. Dunn, S. A. Sunchine, and J. A. Ibers, Physical and structural properties of the new layered compounds $\mathrm{Ta}_{2} \mathrm{NiS}_{5}$ and $\mathrm{Ta}_{2} \mathrm{NiSe}_{5}$, J. Less-Common Met. 116, 51 (1986).

[23] Y. F. Lu, H. Kono, T. I. Larkin, A. W. Rost, T. Takayama, A. V. Boris, B. Keimer, and H. Takagi, Zero-gap semiconductor to excitonic insulator transition in $\mathrm{Ta}_{2} \mathrm{NiSe}_{5}$, Nat. Commun. 8, 14408 (2017).

[24] A. Nakano, K. Sugawara, S. Tamura, N. Katayama, K. Matsubayashi, K. Sugimoto, N. Maejima, A. Machida, T. Watanuki, and H. Sawa, Pressure-induced coherent slidinglayer transition in the excitonic insulator $\mathrm{Ta}_{2} \mathrm{NiSe}_{5}, \mathrm{IUCrJ} \mathbf{5}$, 158 (2018).

[25] K. Seki, Y. Wakisaka, T. Kaneko, T. Toriyama, T. Konishi, N. Katayama, M. Nohara, H. Takagi, T. Mizokawa, and Y. Ohta, Excitonic Bose-Einstein condensation in $\mathrm{Ta}_{2} \mathrm{NiSe}_{5}$ above room temperature, Phys. Rev. B 90, 155116 (2014).

[26] Y. Wakisaka, T. Sudayama, K. Takubo, T. Mizokawa, M. Arita, H. Namatame, M. Taniguchi, N. Katayama, M. Nohara, and H. Takagi, Excitonic Insulator State in $\mathrm{Ta}_{2} \mathrm{NiSe}_{5}$ Probed by Photoemission Spectroscopy, Phys. Rev. Lett. 103, 026402 (2009).

[27] K. Fukutami, R. Stania, J. Jung, E. F. Schwier, K. Shimada, C. I. Kwon, J. S. Kim, and H. W. Yeom, Electrical Tuning of the Excitonic Insulator Ground State of $\mathrm{Ta}_{2} \mathrm{NiSe}_{5}$, Phys. Rev. Lett. 123, 206401 (2019) 
[28] J. Lee, C.-J. Kang, M. J. Eom, J. S. Kim, B. I. Min, and H. W. Yeom, Strong interband interaction in the excitonic insulator phase of $\mathrm{Ta}_{2} \mathrm{NiSe}_{5}$, Phys. Rev. B 99, 075408 (2019).

[29] K. Sugimoto, S. Nishimoto, T. Kaneko, and Y. Ohta, Strong Coupling Nature of the Excitonic Insulator State in $\mathrm{Ta}_{2} \mathrm{NiSe}_{5}$, Phys. Rev. Lett. 120, 247602 (2018).

[30] T. I. Larkin, A. N. Yaresko, D. Pröpper, K. A. Kikoin, Y. F. Lu, T. Takayama, Y. L. Mathis, A. W. Rost, H. Takagi, B. Keimer, and A. V. Boris, Giant exciton Fano resonance in quasi-onedimensional $\mathrm{Ta}_{2} \mathrm{NiSe}_{5}$, Phys. Rev. B 95, 195144 (2017).

[31] T. I. Larkin, R. D. Dawson, M. Höppner, T. Takayama, M. Isobe, Y. L. Mathis, H. Takagi, B. Keimer, and A. V. Boris, Infrared phonon spectra of quasi-one-dimensional $\mathrm{Ta}_{2} \mathrm{NiSe}_{5}$ and $\mathrm{Ta}_{2} \mathrm{NiS}_{5}$, Phys. Rev. B 98, 125113 (2018).

[32] S. Mor, M. Herzog, J. Noack, N. Katayama, M. Nohara, H. Takagi, A. Trunschke, T. Mizokawa, C. Monney, and J. Stähler, Inhibition of the photoinduced structural phase transition in the excitonic insulator $\mathrm{Ta}_{2} \mathrm{NiSe}_{5}$, Phys. Rev. B 97, 115154 (2018).

[33] D. Werdehausen, T. Takayama, G. Albrecht, Y. Lu, H. Takagi, and S. Kaiser, Photo-excited dynamics in the excitonic insulator $\mathrm{Ta}_{2} \mathrm{NiSe}_{5}$, J. Phys.: Condens. Matter 30, 305602 (2018).

[34] S. Mor, M. Herzog, D. Golež, P. Werner, M. Eckstein, N. Katayama, M. Nohara, H. Takagi, T. Mizokawa, C. Monney, and J. Stähler, Ultrafast Electronic Band Gap Control in an Excitonic Insulator, Phys. Rev. Lett. 119, 086401 (2017).

[35] K. Okazaki, Y. Ogawa, T. Suzuki, T. Yamamoto, T. Someya, S. Michimae, M. Watanabe, Y.-F. Lu, M. Nohara, H. Takagi, N. Katayama, H. Sawa, M. Fujisawa, T. Kanai, N. Ishii, J. Itatani, T. Mizokawa, and S. Shin, Photo-induced semimetallic states realised in electron-hole coupled insulators, Nat. Commun. 9, 4322 (2018).

[36] T. Tang, H. Wang, S. Duan, Y. Yang, C. Huang, Y. Guo, D. Qian, and W. Zhang, Non-Coulomb strong electron-hole binding in $\mathrm{Ta}_{2} \mathrm{NiSe}_{5}$ revealed by time- and angle-resolved photoemission spectroscopy, Phys. Rev. B 101, 235148 (2020).

[37] D. Werdehausen, T. Takayama, M. Höppner, G. Albrecht, A. W. Rost, Y. Lu, D. Manske, H. Takagi, and S. Kaiser, Coherent order parameter oscillations in the ground state of the excitonic insulator $\mathrm{Ta}_{2} \mathrm{NiSe}_{5}$, Sci. Adv. 4, eaap8652 (2018).

[38] D. Werdehausen, S. Y. Agustsson, M. Kim, P. Shabestari, E. Huang, A. Pokharel, T. Larkin, A. Boris, T. Takayama, Y. Lu, A. W. Rost, H. Chu, A. Yaresko, M. Höppner, A. Schulz, D. Manske, B. Keimer, and H. Takagi, and S. Kaiser, Ultrafast dynamics and coherent order parameter oscillations under photo-excitation in the excitonic insulator $\mathrm{Ta}_{2} \mathrm{NiSe}_{5}$, Proc. SPIE 10638, Ultrafast Bandgap Photonics III, 1063803 (2018).

[39] P. Andrich, H. M. Bretscher, Y. Murakami, D. Golež, B. Remez, P. Telang, A. Singh, L. Harnagea, N. R. Cooper, A. J. Millis, P. Werner, A. K. Sood, and A. Rao, Imaging the coherent propa- gation of collective modes in the excitonic insulator candidate $\mathrm{Ta}_{2} \mathrm{NiSe}_{5}$ at room temperature, arXiv:2003.10799.

[40] B. Remez and N. R. Cooper, Effects of disorder on the transport of collective modes in an excitonic condensate, Phys. Rev. B 101, 235129 (2020).

[41] Y. Murakami, D. Golež, T. Kaneko, A. Koga, A. J. Millis, and P. Werner, Collective modes in excitonic insulators: Effects of electron-phonon coupling and signatures in the optical response, Phys. Rev. B 101, 195118 (2020).

[42] T. H Nakano, S. Tamura, N. Katayama, S. Tsutsui, and H. Sawa, Antiferroelectric distortion with anomalous phonon softening in the excitonic insulator $\mathrm{Ta}_{2} \mathrm{NiSe}_{5}$, Phys. Rev. B 98, 045139 (2018).

[43] T. Kaneko, T. Toriyama, T. Konishi, and Y. Ohta, Orthorhombic-to-monoclinic phase transition of $\mathrm{Ta}_{2} \mathrm{NiSe}_{5}$ induced by the Bose-Einstein condensation of excitons, Phys. Rev. B 87, 035121 (2013).

[44] G. Mazza, M. Rösner, L. Windgätter, S. Latini, H. Hübener, A. J. Millis, A. Rubio, and A. Georges, Nature of Symmetry Breaking at the Excitonic Insulator Transition: $\mathrm{Ta}_{2} \mathrm{NiSe}_{5}$, Phys. Rev. Lett. 124, 197601 (2020).

[45] M. D. Watson, I. Marković, E. A. Morales, P. Le Fèvre, M. Merz, A. A. Haghighirad, and P. D. C. King, Band hybridization at the semimetal-semiconductor transition of $\mathrm{Ta}_{2} \mathrm{NiSe}_{5}$ enabled by mirror-symmetry breaking, Phys. Rev. Res. 2, 13236 (2020).

[46] A. Subedi, Orthorhombic-to-monoclinic transition in $\mathrm{Ta}_{2} \mathrm{NiSe}_{5}$ due to a zone-center optical phonon instability, Phys. Rev. Mater. 4, 083601 (2020).

[47] See Supplemental Material at http://link.aps.org/supplemental/ 10.1103/PhysRevResearch.2.042039 for details on the experimental methods, the different symmetry channels, laser heating and Bose corrected spectrum.

[48] J. Yan, R. Xiao, X. Luo, H. Lv, R. Zhang, Y. Sun, P. Tong, W. Lu, W. Song, X. Zhu, and Y. Sun, Strong electron-phonon coupling in the excitonic insulator $\mathrm{Ta}_{2} \mathrm{NiSe}_{5}$, Inorg. Chem. 58, 9036 (2019).

[49] P. A. Volkov, M. Ye, H. Lohani, I. Feldman, A. Kanigel, K. Haule, and G. Blumberg, Critical charge fluctuations and quantum coherent state in excitonic insulator $\mathrm{Ta}_{2} \mathrm{NiSe}_{5}$, arXiv:2007.07344.

[50] K. Kim, H. Kim, J. Kim, C. Kwon, J. S. Kim, and B. J Kim, Direct observation of excitonic instability in $\mathrm{Ta}_{2} \mathrm{NiSe}_{5}$, arXiv:2007.08212.

[51] E. Baldini, A. Zong, D. Choi, C. Lee, M. H. Michael, L. Windgaetter, I. I. Mazin, S. Latini, D. Azoury, B. Lv, A. Kogar, Y. Wang, Y. F. Lu, T. Takayama, H. Takagi, A. J. Millis, A. Rubio, E. Demler, and N. Gedik, The spontaneous symmetry breaking in $\mathrm{Ta}_{2} \mathrm{NiSe}_{5}$ is structural in nature, arXiv:2007.02909.

[52] P. Andrich, H. M. Bretscher, P. Telang, A. Singh, L. Harnaga, A. K. Sood, and A. Rao, Ultrafast melting and recovery of collective order in the excitonic insulator $\mathrm{Ta}_{2} \mathrm{NiSe}_{5}$, arXiv:2007.03368. 